\title{
Analysis on Effects of Comprehensive Nursing Management on Control of Respiratory Infections and Nursing Quality
}

\author{
Miaozhu Liu* \\ Department of Respiratory Medicine, General Staff Hospital of Shanxi Coking Coal, Xishan Coal-Electricity Group, \\ Taiyuan, Shanxi 030053, China
}

\begin{abstract}
Objective: To observe and analyze infection control and nursing quality after performing comprehensive nursing management to patients of respiratory diseases. Methods: To conduct a retrospective clinical data analysis of 92 hospitalized patients with respiratory tract infections, who were randomly divided into the study group (46 cases) and control group (46 cases). Both groups were given routine anti-infection treatments. Meanwhile, the study group received comprehensive nursing management, while the control group received routine infection nursing. The clinical effects of patients in the two groups were then compared. Results: There were 45 cases $(7.83 \%)$ of effective clinical infection control in the study group, and 43 cases $(93.48 \%)$ in the control group. No significant difference was observed between the two groups $(p>0.05)$. In terms of significant efficiency, there were 41 cases in the study group $(89.13 \%)$ and 33 cases in the control group (71.74\%) and the difference was statistically significant $(p<$ 0.05). Conclusion: Comprehensive management of respiratory infection in patients under respiratory medication not only controls the infection effectively, but improves nursing quality and clinical effects, and it plays an active role in infection control and nursing quality.
\end{abstract}

\section{KEYWORDS}

Respiratory infections

Comprehensive nursing

Nursing quality

\section{Introduction}

Respiratory tract infection is a common clinical infectious disease which is mostly caused by viruses. It is a disease with strong infection and high incidence. At present, the incidence of respiratory tract infection presents a gradual upward trend. In respiratory medicine, the main infection factors are cross infections, improper use of antimicrobial agents and so forth. To avoid serious effects on patients' physical and mental health, comprehensive management can be given to patients apart from routine anti-infections

\section{Copyright $\odot 2016$ Miaozhu Liu}

doi: $10.18686 /$ jn.v5i4.9

Received: August 30, 2016; Accepted: November 15, 2016;

Published online: December 29, 2016

This is an open-access article distributed under the terms of the Creative Commons Attribution Unported License (http://creativecommons.org/ licenses/by-nc/4.0/), which permits unrestricted use, distribution, and reproduction in any medium, provided the original work is properly cited.

${ }^{*}$ Correspondence author: Department of Respiratory Medicine, General Staff Hospital of Shanxi Coking Coal, Xishan Coal-Electricity Group, Taiyuan, Shanxi 030053, China. Email:liu_miaozhu53@126.com
[1]. 92 cases of patients with respiratory tract infections in the hospital were chosen for this study, and the effects of comprehensive management on infection control and nursing qualities were analyzed.

\section{General data and methods 2.1. General data}

92 cases of inpatients with respiratory tract infections, treated in the respiratory medicine department of our hospital from June 2014 to June 2015 were chosen as research subjects. 51 cases were males and 41 cases were females, aged from 16 to 77 years old. These subjects consisted of 49 cases of bronchitis, 18 cases of bacterial pneumonia and 25 cases of chronic bronchitis.

Inclusion criteria: (1) Patients with a clinical diagnosis of respiratory infections; (2) No liver and kidney function failure; and (3) Patients of respiratory diseases with high blood pressure, diabetes and heart failure. Exclusion criteria: (1) Patients with fatal infectious diseases; (2) Patients who were unable to complete the study smoothly; and (3) Patients who disagreed with this research plan. The 
subjects were divided into the study and control groups at random and there were 46 cases in each group. The study group consisted of 25 males and 21 females with an age range of 16 to 75.26 males and 20 females made up the control group ranging from 18 to 77 years old. The difference in gender, age and pathogenic conditions between the two groups had no statistical significance $(p>0.05)$.

\subsection{Methods}

\subsubsection{Routine anti-infection nursing in the control group}

Patients in the control group were given routine antiinfection treatments and nursing following doctor's advice, such as psychological nursing, health guidance and so forth. Some antibiotics like penicillin, clindamycin and cephalosporin were adopted into the routine anti-infection treatment based on patients' conditions. In addition to the routine anti-infection nursing, observations and patients' improved conditions in coughing, fever, and dry and wet rales in their lungs were recorded.

\subsubsection{Comprehensive nursing management in the study group}

(1) Nursing management systems: Inpatients visiting rate is high in respiratory medicine, which could easily contaminate the air in the ward and increases the chances of picking up pathogenic bacteria. Therefore, the related system of nursing management must be implemented for respiratory medicine. Meanwhile, reasonable visiting times and patient accompanying systems were formulated, and wards were disinfected and ventilated to maintain appropriate humidity [2].

(2) Health education: Respiratory diseases and methods of preventive control were explained to patients and their family in various ways by using brochures, PowerPoint presentations and so forth, which can enhance their selfprotection consciousness and increase their knowledge of the diseases effectively. Patients would understand the significance of reasonable treatment to promote rehabilitation which is beneficial for the improvement of clinical efficacy.

(3) Psychological nursing: Patients were provided with special psychological guidance, encouragement, care and respect. In addition, communication with patients was actively done so as to understand their psychological state, which is beneficial to adopt specific psychological nursing measures. Psychological nursing can develop patients' confidence to overcome the disease and recuperate from illnesses [3].
(4) Rational use of antibacterial agents: Antibacterial agents can promote patients' recovery. In order to avoid patients from developing resistance to drugs due to an irrational use of antibacterial agents, nursing staff examined patients in detail according to their conditions. Drugs were provided based on examination reports and doctors' advice.

\subsection{Evaluation criteria of curative effect}

Remarkable recovery, effective recovery and inefficient recovery were classified as evaluation criteria of patients' treatment and curative effects after nursing. Remarkable recovery was categorized as patients' clinical symptoms were alleviated and lungs regain normalcy after X-ray examinations, effective recovery classified as patients' clinical symptoms were relieved significantly and lung conditions have shown some improvement and inefficient recovery were if patients' clinical symptoms were not alleviated significantly or became worse and there were no significant improvement in lung disease. Remarkable and effective recoveries were regarded as total effective cases.

\subsection{Statistical methods}

All of the clinical data were processed using SPSS 13.0. Measurement materials were analyzed for $t$ tests, measurement data were expressed by $(\bar{x} \pm s)$, and data counts were tested for $x^{2}$ The differences were statistically significant $(p$ $<0.05)$.

\section{Results}

Two groups of patients with respiratory tract infections in the hospital completed treatment and related nursing. In the aspects of clinical infection control, there were 45 total effective cases in the study group which accounted for $97.83 \%$, while there were 43 total effective cases in the control group, accounting for $93.48 \%$. There were no statistical differences between the two groups $(p<0.05)$ (Table 1).

\section{Discussion}

Respiratory medicine is one of the departments receiving a lot of patients in medical institutions. Consequently, patients unknowingly transport many kinds of bacteria and infection occurs from time to time, which has serious impacts on clinical treatments and hampers medical work as well. If patients cannot achieve ideal treatment results, disputes will arise between patients and medical institutions. As a result, reports on conflicts are relatively increasing,

Table 1. Comparison of clinical efficacy between study and control groups [n (\%)].

\begin{tabular}{cccccc}
\hline Group & $\mathrm{n}$ & Remarkable recovery & Effective recovery & Inefficient recovery & Total effective rate (\%) \\
\hline Control & 46 & $33(71.74 \%)$ & $10(21.74 \%)$ & $3(6.52 \%)$ & 93.48 \\
Study & 46 & $41(89.13 \%)$ & $4(8.70 \%)$ & $1(2.17 \%)$ & 97.83 \\
$\chi^{2}$ & - & 4.420 & 3.033 & 1.045 & 1.684 \\
$p$ & - & $<0.05$ & $>0.05$ & $>0.05$ & $>0.05$ \\
\hline
\end{tabular}


damaging the social image of medical institutions. It has become an important issue troubling clinical work. Therefore, the study of respiratory infection control becomes a hot topic within the medical circles.

Respiratory infection is caused by many factors and the main factors include cross infection, improper use of antimicrobial agents and so forth. With infection rates increasing, it is an important task for nursing staff to take reasonable measures to avoid respiratory system infection of patients, which is a reflection of nursing quality as well $[4,5]$. Implementing nursing management system, visitation and company care system as well as regulating nursing behaviors can reduce the rate of patients' respiratory infections effectively. Performing health education to patients and their family can increase their knowledge and awareness of respiratory infection prevention and control. Meanwhile, bacteria and viruses outside of hospitals are less likely to be brought into the hospital by means of visitations. To some extent, it plays an effective role in the prevention and control of the disease. Encouraging and supporting the patients by providing psychological assistance could develop their confidence to overcome the disease by strengthening their psychological defenses. With a more positive attitude, patients' participation is enhanced. Its role cannot be ignored in the control of infection. Furthermore, patients were provided with moderate amounts of antimicrobial agents. At the same time, wards needed to be disinfected and ventilated constantly to preserve a healthier environment. Those measures can control respiratory infections effectively and improve clinical efficacy and patients' quality of life.

Liu Chanhong's intensive study of nursing management effects on the control of respiratory infections have shown that the occurrence rate of infection control in the study group is lower than the control group when patients in the study group were given routine anti-infection treatments as well as nursing management measures. Thus it was concluded that the application of nursing management measures to patients of respiratory infections can effectively improve nursing quality and clinical therapy effects. Consequently, the overall treatment level of the hospital can be improved. It is a solution that could be promoted in clinics. In this study, treatment and related nursing were completed in both groups of patients with respiratory tract infections. There were 45 total effective cases in the study group, covering $97.83 \%$. While 43 total effective cases in the control group, accounted for $93.48 \%$. There were no significant differences between the two groups $(p>0.05)$. In terms of significant efficiency rates of the two groups, the study group was apparently higher than the control group and the difference was statistically significant $(p<0.05)$. The reasons why it was different than the previous studies were due to many factors. On the one hand, routine nursing measures were comprehensive in this research, which covered various aspects of the patients. In addition, patients' resistance to drugs is relatively low and as a result, antibiotics and anti-infective drugs were function fully, improving the effect of infection control. On the other hand, the number of chosen subjects was also relatively small. Only 92 cases of patients with infection of respiratory medicine were selected as subjects in this research, and there existed a certain gap compared with large sample standards. As for the infection control effect, the results in this research is consistent with some existing research, that is, nursing management can control the occurrence of infection effectively, which guarantees the safety of clinical treatment [6-8].

In conclusion, providing routine anti-infection treatment and comprehensive nursing management to inpatients with respiratory tract infections will not only improve patients' understanding of the disease, but also avoid improper use of antibacterial agents effectively, thus achieving the aim of controlling infection and improving nursing quality and clinical effects.

\section{References}

1. Wen HM. Cause analysis of respiratory medicine nosocomial infection and nursing measures. For All Health. 2013;7(6):161.

2. Wang JY, Qu Yi, Li Zhijun. Analysis of nosocomial infection in the internal medicine wards and nursing countermeasures. Hebei Medical Journal. 2009;31(7):880-881.

3. Wu FC. Common infectious pathogen and related factors in respiratory internal medicine. Jilin Medical Journal. 2013;1(28):4959-4960.

4. Jin ZY. The effect of comprehensive nursing management on the control of the infection of respiratory medicine and nursing quality. Today Nurses. 2013;35(4):157-188.

5. Zhang GF. The applied analysis of three-level quality management to reduce hospital infection. Journal of Clinical Medicine in Practice. 2015;21(12):163-165.

6. Mo LL, Liu XX, Zhong YH. Application research of tracking methodology in respiratory medicine the whole nursing patients with severe disease. Medical Innovation of China. 2015;33(14):73-76.

7. Li XH. The effect of PDCA cycle management on reducing complications of patients with respiratory medicine venipuncture. Journal of Qiqihar University of Medicine. 2015;15(4):561-562.

8. Liu $\mathrm{CH}$, Zhang $X$. The effect of nursing management on nosocomial infection in patients with respiratory medicine. World Latest Medicine Information. 2015;10(2):215-216. 